www.nature.com/hdy

\title{
ORIGINAL ARTICLE \\ Decoupling of differentiation between traits and their underlying genes in response to divergent selection
}

\begin{abstract}
A Kremer ${ }^{1,2}$ and V Le Corre ${ }^{3}$
We dissected the relationship between genetic differentiation $\left(Q_{S T}\right)$ for a trait and its underlying genes ( $G_{S T q}$, differentiation for a quantitative locus) in an evolutionary context, with the aim of identifying the conditions in which these two measurements are decoupled. We used two parameters $\left(\theta_{\mathrm{B}}\right.$ and $\left.\theta_{\mathrm{W}}\right)$ scaling the contributions of inter- and intrapopulation allelic covariation between genes controlling the trait of interest. We monitored the changes in $\theta_{\mathrm{B}}$ and $\theta_{\mathrm{W}}, Q_{\mathrm{ST}}$ and $G_{\mathrm{ST} q}$ over successive generations of divergent and stabilizing selection, in simulations for an outcrossing species with extensive gene flow. The dynamics of these parameters are characterized by two phases. Initially, during the earliest generations, differentiation of the trait increases very rapidly and the principal and immediate driver of $Q_{\mathrm{ST}}$ is $\theta_{\mathrm{B}}$. During subsequent generations, $G_{\mathrm{ST} q}$ increases steadily and makes an equal contribution to $Q_{S T}$. These results show that selection first captures beneficial allelic associations at different loci at different populations, and then targets changes in allelic frequencies. The same patterns are observed when environmental change modifies divergent selection, as shown by the very rapid response of $\theta_{\mathrm{B}}$ to the changes of selection regimes. We compare our results with previous experimental findings and consider their relevance to the detection of molecular signatures of natural selection.
\end{abstract}

Heredity (2012) 108, 375-385; doi:10.1038/hdy.2011.81; published online 14 September 2011

Keywords: differentiation; $Q_{S T} ; G_{S T}$; intergenic disequilibria

\section{INTRODUCTION}

The detection of genomic imprints of natural selection has become a major area of research in ecological genetics (Nielsen, 2005; Wright and Gaut, 2006). One of the approaches used is based on the detection of genes displaying higher levels of population differentiation than would be expected under neutral evolution (the so-called 'outlier' loci; Storz, 2005; Holderegger et al., 2008). In most cases, the rationale of 'outlier' detection is based on the existence of a high degree of phenotypic differentiation of the trait, as observed in common garden experiments, suggesting similar levels of differentiation at the genomic level. For example, in forest trees, in which extensive common gardens have been established, known as provenance tests, substantial population differentiation has been observed for almost all phenotypic traits assessed (see Wright (1976) and Morgenstern (1996) for reviews on North American species, and König (2005) for a review on European species). Only a few studies have investigated the differentiation of traits and their underlying genes, and these studies have concluded that there is considerable discrepancy between these two measurements (Hall et al., 2007; Luquez et al., 2007; Namroud et al., 2008). Theoretical predictions supported by simulations have indeed indicated that there may be large discrepancies between phenotypic differentiation $\left(Q_{\mathrm{ST}}\right)$ and differentiation of the genes controlling the trait $\left(G_{\mathrm{ST} q}\right.$, for quantitative loci, as suggested by Santure and Wang (2009), to avoid confusion with neutral $G_{\mathrm{ST}}$ or $\left.F_{\mathrm{ST}}\right)$. Latta $(1998,2004)$ and McKay and Latta (2002) have shown that this discrepancy stems from the intergenic disequilibria accumulated through selection, which decouples differentiation at the trait and gene levels. In a previous study, we investigated the contribution of intergenic disequilibria to overall phenotypic differentiation, theoretically, in more detail (Le Corre and Kremer, 2003). We confirmed that high levels of differentiation could be observed for a trait, with only low levels of differentiation for the genes controlling the trait, and reviewed various evolutionary scenarios under which this discrepancy would be maintained or increased. In extreme cases, such as outcrossing species with large populations and high gene flow undergoing divergent selection, as in trees, there might be no signature at all at individual loci, despite a high degree of phenotypic differentiation. Recent developments along these lines have considered the contribution of dominance to the decoupling of $Q_{\mathrm{ST}}$ and $G_{\mathrm{ST} q}$, (Goudet and Büchi, 2006; Goudet and Martin, 2007; Santure and Wang, 2009). Under divergent selection, the contrast between these two measurements of differentiation increases with dominance (Santure and Wang, 2009). We therefore consider that the additive model used in this study provides the lower limits of decoupling between $Q_{\mathrm{ST}}$ and $G_{\mathrm{ST} q}$ in more complex situations, including gene interactions.

We explore here the progressive decoupling of $Q_{\mathrm{ST}}$ and $G_{\mathrm{ST} q}$ under different evolutionary scenarios. Our previous study focused on the equilibrium values of $Q_{S T}$ and $G_{S T q}$ (Le Corre and Kremer, 2003). Here, we address their transient dynamics over time and consider different genetic architectures of the trait undergoing selection. Interest in the speed of adaptive differentiation in heterogeneous landscapes has increased recently (Kawecki, 2008; Lopez et al., 2008;

${ }^{1}$ INRA, UMR1202 Biodiversité Gènes et Communautés, Cestas, France; ²Université de Bordeaux1, UMR1202 Biodiversité Gènes et Communautés, Talence, France and 3INRA, UMR1210 Biologie et Gestion des Adventices, Dijon F-21065, France

Correspondence: Dr A Kremer, INRA, UMR1202 Biodiversité Gènes et Communautés, 69 route d'Arcachon, 33610 Cestas Cedex, France.

E-mail: kremer@pierroton.inra.fr

Received 22 December 2010; revised 15 June 2011; accepted 27 June 2011; published online 14 September 2011 
Bjorklund et al., 2009). Evolutionary responses are often inferred from the divergence or differentiation of adaptive traits observed in a set of populations undergoing divergent selection (Hendry et al., 2001; Crispo, 2008; Rasanen and Hendry, 2008) or from the molecular divergence of genes of adaptive relevance (Kapralov and Filatov, 2006; Nosil et al., 2009). Furthermore there is an increasing concern about the rate of response of natural populations to ongoing climate change (Aitken et al., 2008). It is therefore timely to reconsider the dynamics of differentiation in an evolutionary context. Our main objective is to monitor changes of $Q_{\mathrm{ST}}$ and $G_{\mathrm{ST} q}$ and their decoupling in silico by considering different divergent selection regimes, including nonequilibrium situations. We used two parameters $\left(\theta_{\mathrm{B}}\right.$ and $\left.\theta_{\mathrm{W}}\right)$ scaling the contributions of between- and within-population disequilibria to $Q_{\text {ST. We highlight the way in which divergent selection generates a }}$ positive $\theta_{\mathrm{B}}$, which becomes in most circumstances the main driver of $Q_{\text {ST }}$. We focus on the particular case of species with large populations and high levels of gene flow, such as trees, in which high levels of trait differentiation are maintained in the presence of high levels of gene flow (Savolainen et al., 2007). We aimed to identify the situations in which the traces of natural selection acting on the genes controlling the trait might be blurred by intergenic disequilibria, thereby limiting the power to detect 'outlier' loci.

\section{MATERIALS AND METHODS}

\section{Decoupling of $Q_{\mathrm{ST}}$ and $G_{\mathrm{ST} q}$}

We focus here on the comparative monitoring of population differentiation of a diploid organism for a phenotypic trait and the underlying genes contributing to the trait, assuming that all these genes are known. The trait is assessed in different populations subject to selection and exchanging genes through gene flow. At any time during the evolutionary process, differentiation of the trait and of the underlying genes can be assessed. In a previous study, we derived a relationship between differentiation for a quantitative trait $\left(Q_{\mathrm{ST}}\right)$ and the mean differentiation of the $n$ loci controlling the trait $\left(G_{\mathrm{ST} q}\right.$; Le Corre and Kremer, 2003) under the following assumptions:

- Diallelic loci for which the two alleles have symmetric additive effects $(+\alpha / 2$ and $-\alpha / 2$ ).

- Equal contributions of the various loci to the additive value of the trait.

- Additive effects of the alleles at each locus, with no consideration of dominance or epistasis.

$$
Q_{\mathrm{ST}}=\frac{\left(1+\theta_{\mathrm{B}}\right) G_{\mathrm{ST} q}}{\left(\theta_{\mathrm{B}}-\theta_{\mathrm{W}}\right) G_{\mathrm{ST} q}+1+\theta_{\mathrm{W}}}
$$

where

$$
\theta_{\mathrm{W}}=\frac{\sum_{i=1}^{n} \sum_{j \neq i}^{n} \operatorname{Cov}_{\mathrm{W} i, j}}{\sum_{i=1}^{n} \sigma_{\mathrm{W} i}^{2}} \text { and } \theta_{\mathrm{B}}=\frac{\sum_{i=1}^{n} \sum_{j \neq i}^{n} \operatorname{Cov}_{\mathrm{B} i, j}}{\sum_{i=1}^{n} \sigma_{\mathrm{B} i}^{2}}
$$

$\sigma_{\mathrm{W} i}^{2}$ is the within-population variance of additive effects at locus $i$ contributing to the trait. At the single-locus level, $\sigma_{\mathrm{W} i}^{2}$ is also called genic variance. The genic variance is assessed in all populations, and in what follows $\sigma_{\mathrm{W} i}^{2}$ is the average value across populations. $\sigma_{\mathrm{B} i}^{2}$ is the variance of population effects of locus $i$.

$\operatorname{Cov}_{W i, j}$ and $\operatorname{Cov}_{B i, j}$ are the within- and between-population covariances of the additive and population effects.

$\theta_{\mathrm{W}}$ and $\theta_{\mathrm{B}}$ are the relative contributions of the genetic covariances between loci to the additive and between-population variances of the trait $\left(V_{\mathrm{W}}\right.$ and $V_{\mathrm{B}}$, respectively), with respect to the single-locus variances $\left(\sigma_{\mathrm{W} i}^{2}\right.$ and $\sigma_{\mathrm{Bi}}^{2}$.

$$
V_{\mathrm{W}}=\left(1+\theta_{\mathrm{W}}\right) \sum_{i=1}^{n} \sigma_{\mathrm{W} i}^{2} \quad \text { and } \quad V_{\mathrm{B}}=\left(1+\theta_{\mathrm{B}}\right) \sum_{i=1}^{n} \sigma_{\mathrm{B} i}^{2}
$$

$V_{\mathrm{W}}$ is the additive variance within populations.
$V_{\mathrm{B}}$ is the between-population variance.

According to (1), $Q_{\mathrm{ST}}$ is a monotone increasing function of $G_{\mathrm{ST} q}$ and $\theta_{\mathrm{B}}$, and a monotone decreasing function of $\theta_{\mathrm{W}}$, reflecting the contrasting contributions of the within- and between-population disequilibria to $Q_{\mathrm{ST}}$. Interpopulation disequilibria between loci tend to increase phenotypic differentiation between populations, whereas intrapopulation disequilibria decrease differentiation.

Relationship (1) holds even when gene flow and selection are not at equilibrium. It is an 'instantaneous' relationship describing the architecture of $Q_{S T}$ in terms of single-locus components $\left(G_{S T q}\right)$ and multilocus components $\left(\theta_{\mathrm{B}}\right.$ and $\left.\theta_{\mathrm{W}}\right)$ at any time through an evolutionary scenario. This relationship also holds for all types and strengths of selection within or between populations and contributing to the divergence between populations.

We can now consider the difference between trait differentiation and gene differentiation by rewriting (1) in a different way:

$$
\frac{Q_{\mathrm{ST}}}{G_{\mathrm{ST} q}}=\frac{\left(1+\theta_{\mathrm{B}}\right)}{\left(\theta_{\mathrm{B}}-\theta_{\mathrm{W}}\right) G_{\mathrm{ST} q}+1+\theta_{\mathrm{W}}}
$$

$$
\left(1+\theta_{\mathrm{B}}\right)>\left(\theta_{\mathrm{B}}-\theta_{\mathrm{W}}\right) G_{\mathrm{ST} q}+1+\theta_{\mathrm{W}} \quad \text { if } \theta_{\mathrm{B}}>\theta_{\mathrm{W}}
$$

From (4) and (5), we can conclude that:

$$
\begin{aligned}
& \text { When } \theta_{\mathrm{B}}=\theta_{\mathrm{W}} \text {, then } Q_{\mathrm{ST}}=G_{\mathrm{ST} q} \\
& \text { When } \theta_{\mathrm{B}}>\theta_{\mathrm{W}} \text {, then } Q_{\mathrm{ST}}>G_{\mathrm{ST} q} \\
& \text { When } \theta_{\mathrm{B}}<\theta_{\mathrm{W}} \text {, then } Q_{\mathrm{ST}}<G_{\mathrm{ST} q}
\end{aligned}
$$

There is no simple relationship between $Q_{\mathrm{ST}}$ and $G_{\mathrm{ST} q}$ in more complex situations, with unequal contributions of loci and multiallelism. However, we used simulations to determine whether relationship (1) was applied in such situations (Le Corre and Kremer, 2003; Figure 3). As expected, the observed differentiation of the trait $\left(Q_{\mathrm{ST}}\right)$ in the case of multiallelism and an unequal contribution of loci were larger than that predicted by Equation (1), but the difference was moderate when $Q_{\text {ST }}$ was below 0.40 . These comparisons suggest that the decoupling of $Q_{\mathrm{ST}}$ and $G_{\mathrm{ST} q}$ would be even more pronounced in these more realistic situations. Hence, relationship (1) is a rather conservative figure, corresponding to the minimum observable discrepancy between $Q_{\mathrm{ST}}$ and $G_{\mathrm{ST} q}$. Similarly, when dominance is taken into account, the difference between the two measurements of differentiation increases with divergent selection and higher levels of dominance (Santure and Wang, 2009). Furthermore, inequalities (6) were also maintained when multiple alleles and unequal contributions of loci were considered in simulations (Le Corre and Kremer, 2003, Figure 4). From the inequalities in (6), we can conclude that the comparison of disequilibria between and within populations can be used to predict the level of decoupling of $G_{\mathrm{ST} q}$ and $Q_{\mathrm{ST}}$.

We further dissected $\theta_{\mathrm{B}}$ and $\theta_{\mathrm{W}}$ taking into account the genetic architecture of the trait, that is, the number of loci contributing to the trait. Within- and between-population genic variances can be expressed in terms of diversity and differentiation parameters in the case of biallelic loci having symmetrical additive effects (Le Corre and Kremer, 2003):

$$
\sigma_{\mathrm{W} i}^{2}=H_{\mathrm{S} i}\left(1+F_{i}\right) \alpha_{i}^{2}
$$

$$
\sigma_{\mathrm{B} i}^{2}=2 D_{\mathrm{ST} i} \alpha_{i}^{2}
$$

where $H_{s i}$ is the mean within-population diversity for locus $i, F_{i}$ the mean within-population fixation index for locus $i$, and $D_{\mathrm{ST} i}$ is the differentiation among populations for locus $i$ (Nei, $1987 D_{\mathrm{ST} i}=H_{T i}-H_{S i}, H_{T i}$ being the total diversity in the metapopulation).

Covariances between allelic effects of loci $i$ and $j$ can be decomposed in terms of disequilibria and allelic effects (Weir, 1990; Hamilton and Cole, 2004) as:

$$
\operatorname{Cov}_{\mathrm{W} i, j}=2 \alpha_{i} \alpha_{j} \Delta_{\mathrm{w} i, j}
$$

$$
\operatorname{Cov}_{B i, j}=4 \alpha_{i} \alpha_{j} \Delta_{\mathrm{Bi}, j} \quad \text { with } \quad \Delta_{\mathrm{B} i, j}=\operatorname{Cov}\left(p_{i}, p_{j}\right)
$$

where $\Delta_{W i, j}$ is the mean composite genotypic disequilibrium (within population) between loci $i$ and $j$ according to Weir, ( $\Delta_{A B}$ in Weir's notation), and 
$\operatorname{Cov}\left(p_{i}, p_{j}\right)$ is the covariance between frequencies $p_{i}$ (allele of positive effect at locus $i$ ) and $p_{j}$ (allele of positive effect at locus $j$ ) calculated across all populations.

If we further assume that the $\alpha_{i}$ and $F_{i}$ values are the same for all loci, and taking $H_{\mathrm{S}}$ as the mean intrapopulation gene diversity over all loci, then $\theta_{\mathrm{W}}$ becomes:

$$
\theta_{\mathrm{W}}=\frac{2(n-1) \Delta_{\mathrm{W}}}{(1+F) H_{\mathrm{S}}}
$$

Similarly assuming that $D_{\mathrm{ST}}$ is the mean differentiation across all loci and that allelic effects are the same across loci, then $\theta_{\mathrm{B}}$ becomes:

$$
\theta_{\mathrm{B}}=\frac{2(n-1) \Delta_{\mathrm{B}}}{D_{\mathrm{ST}}}
$$

where $n$ is the number of loci, and $\Delta_{\mathrm{W}}$ and $\Delta_{\mathrm{B}}$ are the mean values of $\Delta_{\mathrm{W} i, j}$ and $\Delta_{\mathrm{B} i, j}$ over all pairs of loci. As a result, $\theta_{\mathrm{B}}$ and $\theta_{\mathrm{W}}$ are now written in terms of disequilibria and diversity parameters, which can be estimated in natural populations. Expressions (11) and (12) show that $\theta_{\mathrm{B}}$ and $\theta_{\mathrm{W}}$ are dependent on the number of loci contributing to the trait. The decoupling of $Q_{\mathrm{ST}}$ and $G_{\mathrm{ST} q}$ can now be expressed as a function of the number of loci by replacing in (4) $\theta_{\mathrm{W}}$ and $\theta_{\mathrm{B}}$ by their expressions given by (11) and (12)

$$
\frac{Q_{\mathrm{ST}}}{G_{\mathrm{ST} q}}=\frac{(1+x B)}{(B-W) x G_{\mathrm{ST} q}+1+x W}
$$

where $x=2(n-1)$ and $B=\Delta_{\mathrm{B}} / D_{\mathrm{ST}}$ and $W=\Delta_{\mathrm{W}} /(1+F) H_{\mathrm{S}}$ It can be shown that (13) is an increasing function of $x$ when $B>\mathrm{W}$ (or when $\theta_{\mathrm{B}}>\theta_{\mathrm{W}}$ ) and a decreasing function of $x$ when $B<W$ (or when $\theta_{\mathrm{B}}<\theta_{\mathrm{W}}$ ).

\section{Simulations}

We monitored the changes in $\theta_{\mathrm{B}}, \theta_{\mathrm{W}}, G_{\mathrm{ST} q}$ and $Q_{\mathrm{ST}}$ under different evolutionary scenarios, assessing the decoupling of $G_{\mathrm{ST} q}$ and $Q_{\mathrm{ST}}$ over successive generations. Individual-based simulations were carried out with large populations and extensive gene flow, subject to divergent selection. Details of the METAPOP simulation model are provided in Le Corre and Kremer (2003). We considered a phenotypic trait controlled by 5,10 or 30 unlinked loci, each with 20 allelic states. The mutation rate was set at $10^{-5}$ per locus and followed a k-allele model. The genetic value of the trait was the sum of allelic effects over all loci. The additive value at each allele was drawn at random from a Gaussian distribution. The environmental contribution to the phenotypic value of the trait was considered as a Gaussian variable of mean 0 and variance 1 . The variance of the distribution of allelic effects was adjusted, so that the initial genetic variance within populations was equal to 5 (implying that the trait's heritability was initially 0.833 ). These starting values were selected, so that they match with estimates of heritabilities in extant progeny tests (Kremer, 1994); other initial values were tested as well, but the evolution in $\theta_{\mathrm{B}}, \theta_{\mathrm{W}}, G_{\mathrm{ST} q}$ and $Q_{S T}$ showed similar patterns. Throughout the simulations, covariance components of $\theta_{\mathrm{B}}$ and $\theta_{\mathrm{W}}$ were numerically calculated as differences between genetic variances ( $V_{\mathrm{B}}$ and $V_{\mathrm{W}}$, respectively) and genic variances $\left(\sigma_{\mathrm{B} i}^{2}\right.$ and $\sigma_{\mathrm{W} i}^{2}$, respectively) according to Equation (3). Hence, they comprise all sources of allelic associations, for example, between different loci arising on a same gamete (linkage disequilibrium stricto sensu) or of different gametic origins (because of Hardy-Weinberg disequilibrium).

We considered 25 populations, each of size 500, with equal gene flow between all pairs of populations (island model with $\mathrm{Nm}=10$ ). The 25 populations were displayed on a $5 \times 5$ grid system with one-dimensional environmental variation, such that the optimal phenotypic value $Z_{\mathrm{OPT}(k)}$ for the trait in population $k$ followed a cline of variation across the metapopulation. These settings (initial genetic variance and environmental variance within populations, mutation rate, number of populations, population size and migration rate) were kept constant across all simulations.

We focused on the effects of different selection pressures on population differentiation. We considered Gaussian stabilizing selection within each population $k$ toward the local optimal phenotypic value $Z_{\mathrm{OPT}(k)}$. The fitness $W(Z)$ of an individual with phenotype $Z$ located in population $k$ is given by:

$$
W(Z)=\exp \left[-\left(Z-Z_{\mathrm{OPT}(k)}\right)^{2} / 2 \omega^{2}\right]
$$

Table 1 Evolutionary scenarios (a) starting from mutation-migrationdrift equilibrium and (b) with changing selection regimes

(a)

Scenario

Generation 1-3000

\begin{tabular}{lrc}
\hline & $\omega^{2}$ & VarZ \\
1 & 5 & 1 \\
2 & 5 & 5 \\
3 & 50 & 1 \\
4 & 50 & 5 \\
\hline
\end{tabular}

(b)

Scenario

Phase $A$

Phase $B$

Generation 1-100

Generation 101-200

$\omega^{2} \quad \operatorname{var} Z_{O P T}$

$\omega^{2} \quad \operatorname{var} Z_{O P T}$

Uniform selection to strong divergent selection

$\begin{array}{rrrrr}5 & 5 & 0 & 5 & 5 \\ 6 & 50 & 0 & 50 & 5\end{array}$

Moderate divergent to strong divergent selection

$7 \quad 5 \quad 1 \quad 505$

$\begin{array}{llll} & 50 & 1 & 50\end{array}$

Strong divergent to uniform selection

$\begin{array}{lrrrr}9 & 5 & 5 & 5 & 0 \\ 10 & 50 & 5 & 50 & 0\end{array}$

Strong divergent to moderate selection

$\begin{array}{rrrrr}11 & 5 & 5 & 5 & 1 \\ 12 & 50 & 5 & 50 & 1\end{array}$

The strength of within-population selection was scaled by $\omega^{2}$, the selection intensity. We considered two cases with contrasting reported values of $\omega^{2}$ (Roff, 2002): low selection intensity $\left(\omega^{2}=50\right)$ and high selection intensity $\left(\omega^{2}=5\right)$.

Divergent selection was introduced into this setting by the linear gradient of $Z_{\mathrm{OPT}}$ values. On the $5 \times 5$ grid system, this resulted in the assignment of $-x,-x /$ 2, $0, x$ and $x / 2$ as $Z_{\mathrm{OPT}}$ values for the five populations in a single row. The level of divergent selection was scaled by $\operatorname{Var} Z_{\mathrm{OPT}}$, the variance of $Z_{\mathrm{OPT}}$ values between populations $\left(\operatorname{Var} Z_{\mathrm{OPT}}=x^{2} / 2\right)$. Again, we considered two contrasting cases: low levels of divergent selection $\left(\operatorname{Var} Z_{\mathrm{OPT}}=1\right)$ and moderate divergent selection $\left(\operatorname{Var} Z_{\mathrm{OPT}}=5\right)$. In total, we investigated four different selection scenarios combining different selection regimes (Table 1a). At the starting point of the simulations (generation 0 ), we assumed that the 25 populations were at mutation-migration-drift equilibrium (for $\mathrm{Nm}=10$ and $G_{\mathrm{ST} q}=2.4 \%$ ). Hence, some population differentiation for the phenotypic trait existed at time zero, purely due to the neutral differentiation of allelic frequencies $\left(Q_{\mathrm{ST}}=2.4 \%\right)$, but $\theta_{\mathrm{B}}$ and $\theta_{\mathrm{W}}$ were zero at the starting point. The number of alleles initially present at each locus in the metapopulation was fixed at six, according to Ewens' approximate sampling formula (Ewens, 1972). Overall allelic frequencies at each locus were drawn form a Dirichlet distribution with parameter 1. Allelic frequencies for the different populations were then drawn from Dirichlet distributions with parameters equal to the overall frequency scaled by $4 \mathrm{Nm}$ (Beaumont and Balding, 2004). For each scenario, we considered different genetic architectures of the trait undergoing selection, by imposing that the trait was controlled by 5, 10 or 30 loci. A total of 50 replicates (for each combination scenario-number of loci) were run to account for stochastic variation of the various parameters assessed. These simulations were run up to 3000 generations in order to compare equilibrium values, but their dynamics were monitored in detail during the very first 100 generations.

We further investigated additional scenarios aiming at monitoring the decoupling of $G_{\mathrm{ST} q}$ and $Q_{\mathrm{ST}}$ under more realistic situations, where selection 
takes place while some sort of selection was already acting. This was done by subdividing each scenario into two successive phases: phase A from generation 0 to 100 , followed by phase B from generation 101 to 200 (Table 1b).
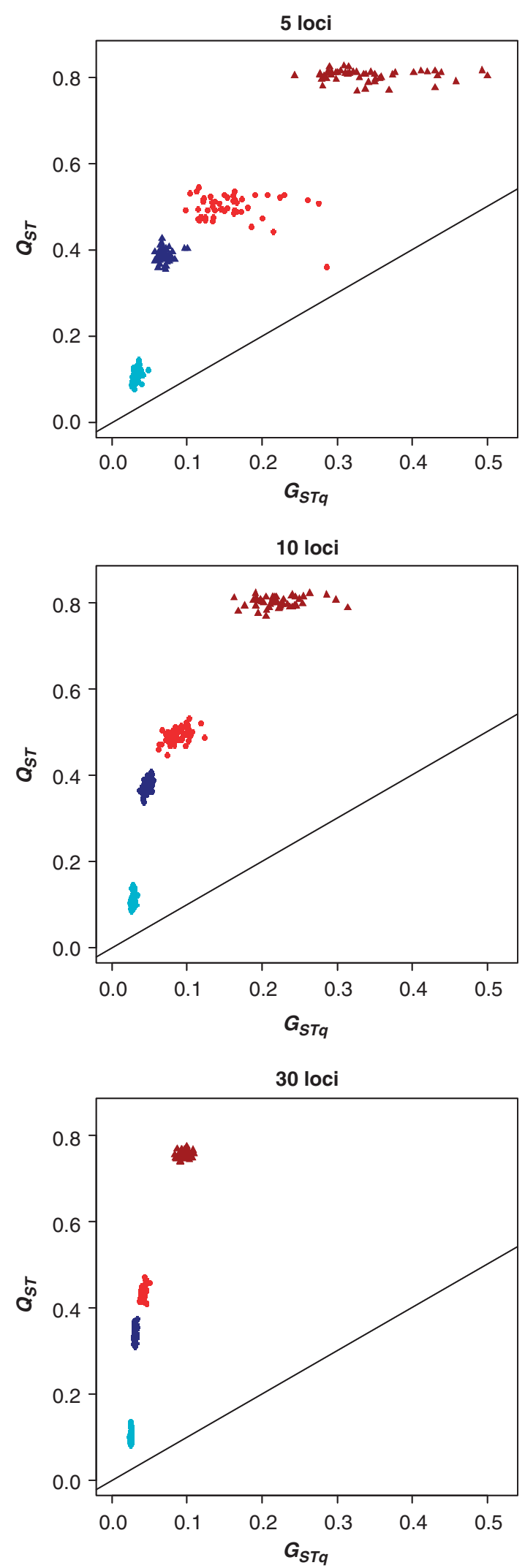

Differences in phases A and B consisted essentially in different levels of divergent selection (uniform, moderate divergent and strong divergent). The rationale for subdividing each scenario into two successive phases was twofold:

(1) to evaluate the impact of different initial situations on the dynamics of the parameters $\left(\theta_{\mathrm{B}}, \theta_{\mathrm{W}}, G_{\mathrm{ST} q}\right.$ and $\left.Q_{\mathrm{ST}}\right)$ by considering nonequilibrium situations as initial states and

(2) to monitor how differentiation of traits and genes respond to sudden changes in selection regimes (either increasing levels or decreasing levels of divergent selection).

\section{RESULTS}

Genetic architecture and decoupling of $G_{\mathrm{ST} q}$ and $Q_{\mathrm{ST}}$

Asymptotic values observed for $G_{\mathrm{ST} q}$ and $Q_{\mathrm{ST}}$ after 3000 generations confirm theoretical predictions that the decoupling between $G_{\mathrm{ST} q}$ and $Q_{\mathrm{ST}}$ is inflated by differences between $\theta_{\mathrm{B}}$ and $\theta_{\mathrm{W}}$ (Figure 1 ). The largest decoupling was observed under strong divergent and stabilizing selection, whereas low divergent and stabilizing selection contributed to moderate decoupling. One of the most striking findings of the simulations was the contrasting response of $\theta_{\mathrm{W}}, \theta_{\mathrm{B}}$ and $G_{\mathrm{ST} q}$ to variation of the number of loci controlling the trait (Table 2 and Figure 1). By contrast, the genetic parameters $V_{\mathrm{W}}, V_{\mathrm{B}}$ and $Q_{\mathrm{ST}}$ remained constant (Supplementary Table 1), except under relaxed selection regimes (scenario 3), where higher levels of $V_{\mathrm{W}}$ and $V_{\mathrm{B}}$ were maintained when the number of loci was larger. As $\theta_{\mathrm{W}}, \theta_{\mathrm{B}}$ and $G_{\mathrm{ST} q}$ are the only components of $Q_{\mathrm{ST}}$ (Equation (1)), there must be some trade-off between $\theta_{\mathrm{W}}, \theta_{\mathrm{B}}$ and $G_{\mathrm{ST} q}$ to maintain $Q_{\mathrm{ST}}$ constant. As predicted by theory (Equations (11) and (12)), $\theta_{\mathrm{W}}$ and $\theta_{\mathrm{B}}$ increase with the number of loci (Table 2). Interestingly, the increase of $\theta_{\mathrm{B}}$ as a

Table 2 Asymptotic values of (a) $\theta_{W}$ and (b) $\theta_{B}$

\begin{tabular}{|c|c|c|c|c|}
\hline \multicolumn{5}{|l|}{ (a) } \\
\hline$\omega^{2}$ & 50 & 50 & 5 & 5 \\
\hline $\operatorname{VarZ}_{O P T}$ & 1 & 5 & 1 & 5 \\
\hline \multicolumn{5}{|l|}{$\mathrm{N}$ loci } \\
\hline 5 & 0.0042 & 0.0394 & 0.0449 & 0.2225 \\
\hline 10 & 0.0066 & 0.0480 & 0.0716 & 0.4027 \\
\hline 30 & -0.0004 & 0.0505 & 0.0748 & 0.4520 \\
\hline \multicolumn{5}{|l|}{ (b) } \\
\hline$\omega^{2}$ & 50 & 50 & 5 & 5 \\
\hline $\operatorname{Var} Z_{O P T}$ & 1 & 5 & 1 & 5 \\
\hline \multicolumn{5}{|l|}{ N loci } \\
\hline 5 & 0.9009 & 1.2824 & 0.7269 & 0.8523 \\
\hline 10 & 1.8024 & 2.9922 & 1.7264 & 2.0447 \\
\hline 30 & 3.1592 & 8.7512 & 6.6065 & 6.9316 \\
\hline
\end{tabular}

Figure 1 Distribution of asymptotic values of $G_{S T q}$ and $Q_{S T}$ under different strengths of divergent and stabilizing selection and with different numbers of loci. Each point represents one stochastic repetition of simulations (50 repetitions for each evolutionary scenario described in Table 1a). Light blue circles: scenario $3, \omega^{2}=50$ and $\operatorname{Var} Z_{\mathrm{OPT}}=1$, weak stabilizing and moderate divergent selection. Dark blue circles: scenario $4, \omega^{2}=50$ and $\operatorname{Var} Z_{\mathrm{OPT}}=5$, weak stabilizing and strong divergent selection. Light red triangles: scenario 1 , $\omega^{2}=5$ and $\operatorname{Var} Z_{\mathrm{OPT}}=1$, strong stabilizing and moderate divergent selection. Dark red triangles: scenario $2, \omega^{2}=5$ and $\operatorname{Var} Z_{\mathrm{OPT}}=5$, strong stabilizing and strong divergent selection 
function of the number of loci fits to the prediction in the simplified diallelic locus case (assuming $\Delta_{\mathrm{B}}$ and $D_{\mathrm{ST}}$ constant). $G_{\mathrm{ST} q}$ decreased with increasing number of loci (Figure 1 and Supplementary Table 1). As predicted by (13), the level of the decoupling $\left(Q_{\mathrm{ST}} / G_{\mathrm{ST} q}\right)$ increased as the number of loci increased (when $\theta_{\mathrm{B}}>\theta_{\mathrm{W}}$ ). A high degree of decoupling of $Q_{\mathrm{ST}}$ and $G_{\mathrm{ST} q}$ was therefore expected when a large number of genes contributed to the trait, as illustrated in Figure 1.

These observations can be extended to the case in which the number of loci increases indefinitely (infinitesimal model), resulting to very low levels of $G_{\mathrm{ST} q}$, and $\theta_{\mathrm{B}}$ becoming the key component of $Q_{\mathrm{ST}}$. A basic assumption of the infinitesimal model is indeed that the allelic frequencies of quantitative loci do not change across generations and that all the between-population variance stems from the intergenic disequilibrium $\left(\theta_{\mathrm{B}}\right.$; Bulmer (1980)).

Figure 1 also illustrates the variation of $Q_{\mathrm{ST}}$ and $G_{\mathrm{ST} q}$ values between stochastic repetitions of a given evolutionary scenario. As the number of genes contributing to the trait decreases, the variation of $G_{\mathrm{ST} q}$ increases over the 50 stochastic repetitions. $Q_{\mathrm{ST}}$ is much less variable than $G_{\mathrm{ST} q}$ across stochastic repetitions. Given the high degree of heterogeneity of $G_{\mathrm{ST} q}$ values, there may be considerable variation of the decoupling of $G_{\mathrm{ST} q}$ and $Q_{\mathrm{ST}}$ for a given setting of $\operatorname{Var} Z_{\mathrm{OPT}}$ and $\omega^{2}$. However, this variation occurs mostly in situations of small numbers of loci and a high intensity of stabilizing selection (Figure 1).

\section{Dynamics of genetic variances and covariances}

We first monitored the dynamics of $\theta_{\mathrm{W}}, \theta_{\mathrm{B}}$ and corresponding variances under selection regimes following an initial mutationmigration-drift equilibrium (scenarios 1-4), considering a trait controlled by 10 loci (Figure 2). In the first few generations $(<10)$ following the induction of selection, the intergenic linkage disequilibrium component $\theta_{\mathrm{W}}$ decreases rapidly. This decrease becomes stronger as the strength of stabilizing selection increases (Figures $2 \mathrm{e}$ and g). These trends follow the predictions of Bulmer $(1974,1980)$, who showed that negative covariances are generated by directional selection in a single population. Our results suggest a similar trend for a subdivided population. Selection induces negative covariance at each generation. However, this covariance is halved by recombination at each generation. Further, the negative covariance component added in each generation decreases steadily because stabilizing selection erodes the genetic variance itself (Figure 3 ). After reaching its minimum, $\theta_{\mathrm{W}}$ begins to increase, possibly even approaching positive values when divergent selection is strong (Figures $2 c$ vs $2 a$; Figures $2 g$ vs $2 e$; Table $2 \mathrm{a}$ ). We suspect that the construction of a positive $\theta_{\mathrm{W}}$ results from an interaction between gene flow and divergent selection. As first observed by Nei and Li (1973), when divergent selection operates in a subdivided population such that allelic frequencies differ between subpopulations, migration produces permanent linkage disequilibrium in each subpopulation. Kirkpatrick et al. (2002; formula 32) showed that the disequilibrium component contributed by migration in each population is a function of the product, across loci, of the differences in allele frequencies between the local population and the migrant pool, and increases with increasing migration rate. As strong divergent selection favors different allelic associations in different populations, migration continually assembles different allelic combinations, thus creating positive disequilibrium within populations. Indeed, our simulations show that divergent selection has a positive effect on $\theta_{\mathrm{W}}$ due to the restoration of positive allelic covariances (Figures 2c and g and Table 2a), as predicted by Nei and Li (1973) and Kirkpatrick et al. (2002).

$\theta_{\mathrm{B}}$ increases very rapidly during the first 5 to 10 generations, particularly under highly divergent selection (Figures $2 \mathrm{~d}$ and $\mathrm{h}$ ). $\theta_{\mathrm{B}}$
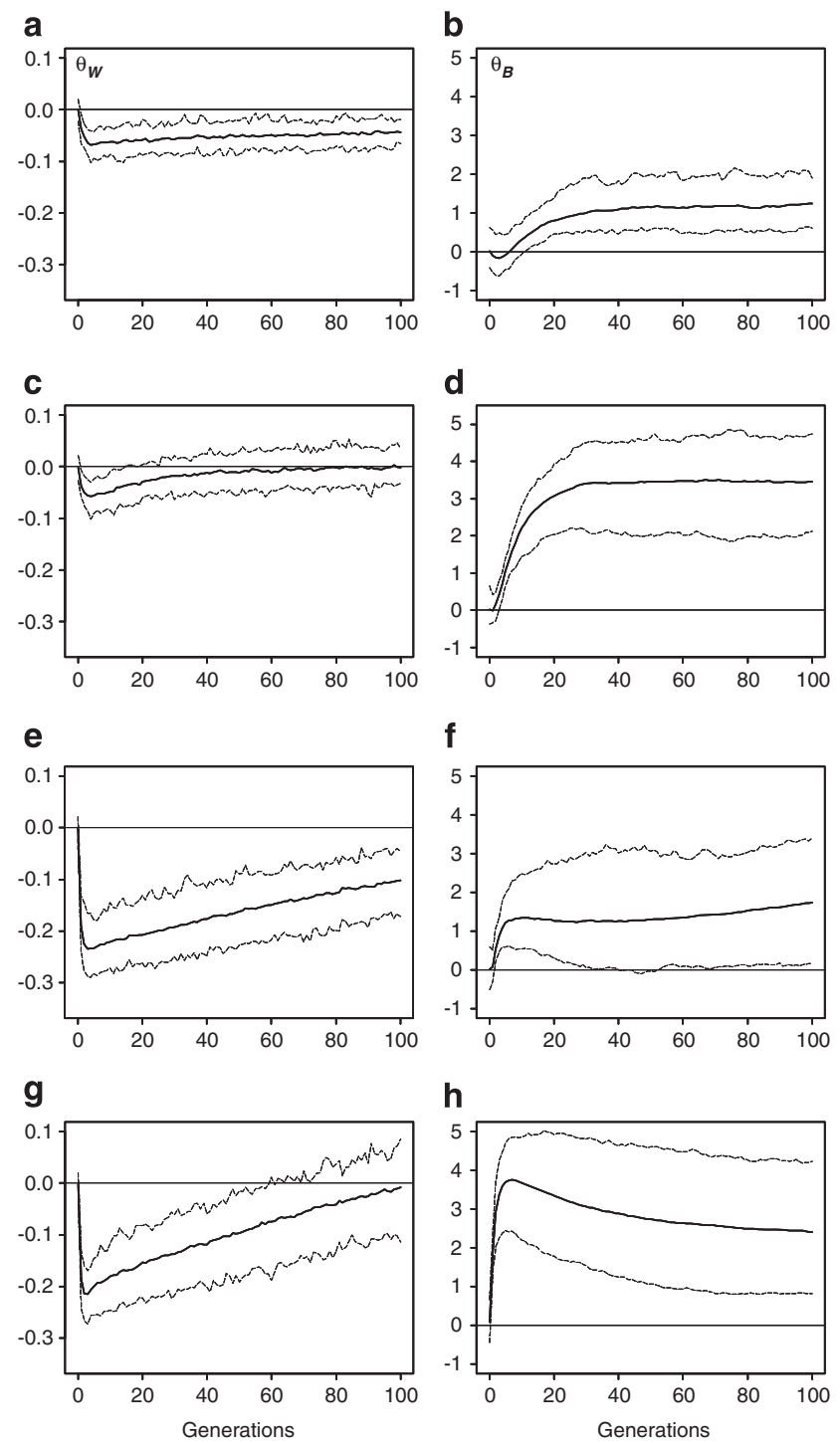

Figure 2 Dynamics of $\theta_{\mathrm{W}}$ and $\theta_{\mathrm{B}}$ over successive generations in response to different selection regimes in the case of 10 loci contributing to the trait. Panels $\mathbf{a}, \mathbf{c}, \mathbf{e}, \mathbf{g}$ correspond to the variation of $\theta_{\mathrm{W}}$ and panels $\mathbf{b}, \mathbf{d}, \mathbf{f}, \mathbf{h}$ correspond to the variation of $\theta_{\mathrm{B}}$. $(\mathbf{a}, \mathbf{b})$ Scenario $3, \omega^{2}=50$ and $\operatorname{Var} Z_{\mathrm{OPT}}=1$, weak stabilizing and moderate divergent selection, (c, d) scenario $4, \omega^{2}=50$ and $\operatorname{Var} Z_{\mathrm{OPT}}=5$, weak stabilizing and strong divergent selection, $(\mathbf{e}, \mathbf{f})$ scenario $1, \omega^{2}=5$ and $\operatorname{Var} Z_{\mathrm{OPT}}=1$, strong stabilizing and moderate divergent selection, $(\mathbf{g}, \mathbf{h})$ scenario $2, \omega^{2}=5$ and $\operatorname{Var} Z_{\mathrm{OPT}}=5$, strong stabilizing and strong divergent selection, 50 repetitions of simulations were conducted for each scenario, bold lines indicate mean values and dashed lines encompass $95 \%$ of the values.

builds up to compensate for the lag between the variance of optimal values $\operatorname{Var} Z_{\mathrm{OPT}}$ and the existing between-population variance $\left(V_{\mathrm{B}}\right)$. At the very beginning (generation 0 ), the only source of $V_{\mathrm{B}}$ is the neutral subdivision of the populations induced by gene flow:

$$
V_{\mathrm{B}_{0}}=2 G_{\mathrm{ST}_{0}} \sigma_{0}^{2}
$$

where $\sigma_{0}^{2}$ is the initial genetic variance in the whole metapopulation and $G_{\mathrm{ST}_{0}}$ is the neutral differentiation. Thus, in the case of a species with low migration, the between-population variance that exists before the onset of divergent selection may be much lower than the variance of optimal values. 
a
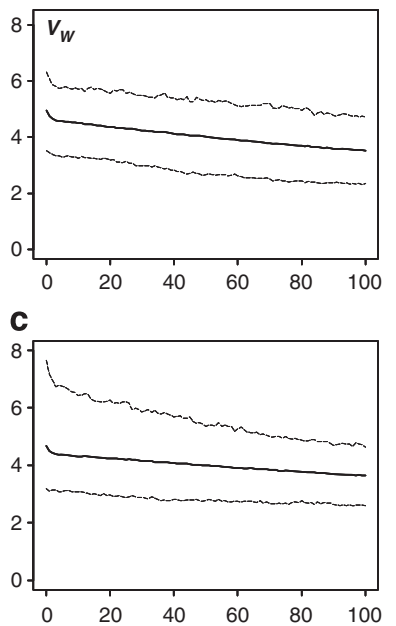

e

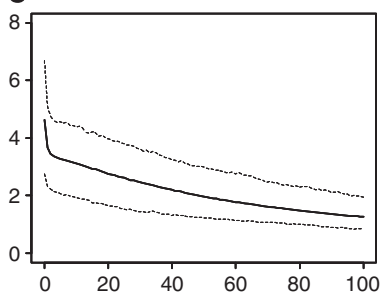

g

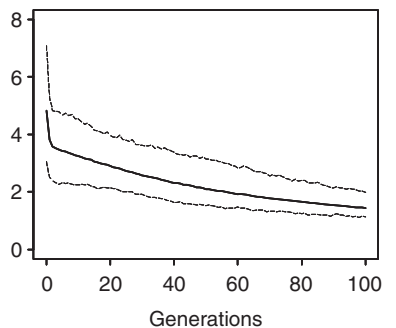

b

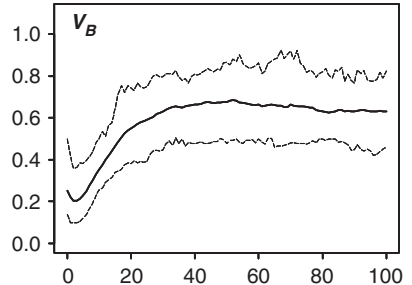

d

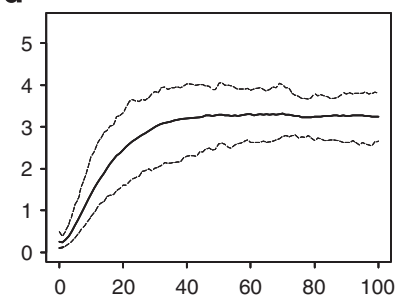

f

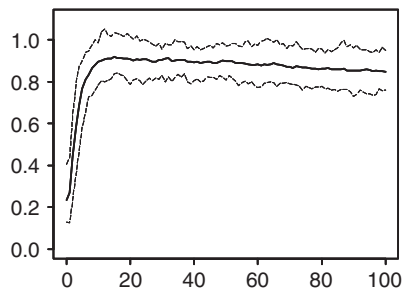

h

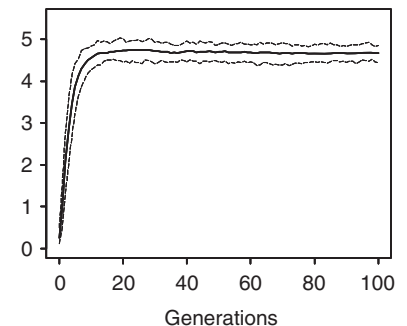

Figure 3 Dynamics of $V_{W}$ and $V_{B}$ over successive generations in response to different selection regimes in the case of 10 loci contributing to the trait. Panels $\mathbf{a}, \mathbf{c}, \mathbf{e}, \mathbf{g}$ correspond to the variation of $V_{W}$ and panels $\mathbf{b}, \mathbf{d}, \mathbf{f}, \mathbf{h}$ correspond to the variation of $V_{\mathrm{B}}$. $(\mathbf{a}, \mathbf{b})$ Scenario $3, \omega^{2}=50$ and $\operatorname{Var} Z_{\mathrm{OPT}}=1$, weak stabilizing and moderate divergent selection, (c, d) scenario $4, \omega^{2}=50$ and $\operatorname{Var} Z_{\mathrm{OPT}}=5$, weak stabilizing and strong divergent selection, $(\mathbf{e}, \mathbf{f})$ scenario $1, \omega^{2}=5$ and $\operatorname{Var} Z_{\mathrm{OPT}}=1$, strong stabilizing and moderate divergent selection, (g,h) scenario $2, \omega^{2}=5$ and $\operatorname{Var} Z_{\mathrm{OPT}}=5$, strong stabilizing and strong divergent selection, 50 repetitions of simulations were conducted for each scenario, bold lines indicate mean values and dashed lines encompass $95 \%$ of the values.

Thereafter, $\theta_{\mathrm{B}}$ is maintained at the same level, or decreases slowly under highly divergent selection (Figure $2 \mathrm{~h}$ ). Thus, simulation results show that changes in genetic covariances both within and between populations occur in the first few generations of selection.

Shortly after $\theta_{\mathrm{B}}$ reaches its peak value, the genetic variance between populations, $V_{\mathrm{B}}$, also attains its maximum value (Figure 3 ), decreasing steadily thereafter. The rate of decrease is stronger when stabilizing selection is weak. It is difficult to detect these dynamics over short time scales (Figure 3), but they are obvious when comparing the asymptotic values of $V_{\mathrm{B}}$ (Supplementary Table 1) with the values of this parameter over the first 100 generations (Figure 3). As alleles are purged from populations, intergenic allelic associations are limited (decay of $\theta_{\mathrm{B}}$; Figures $2 \mathrm{f}$ and $\mathrm{h}$ ) and $V_{\mathrm{B}}$ depends principally on the

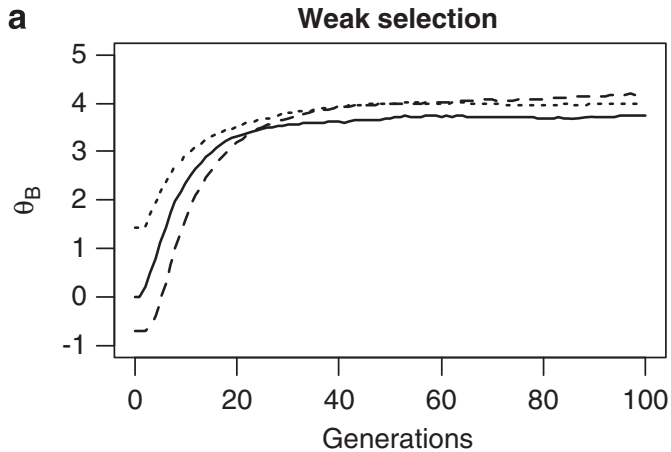

b Strong selection

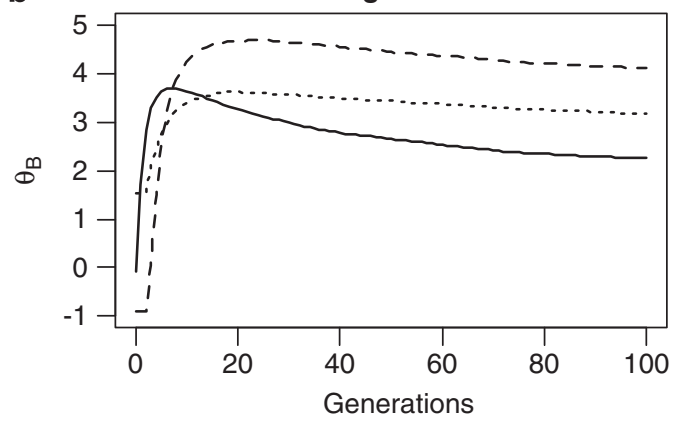

Figure 4 Response of $\theta_{\mathrm{B}}$ to divergent selection $\left(\operatorname{Var} Z_{\mathrm{OPT}}=5\right)$ when (a) selection intensity is weak $\left(\omega^{2}=50\right)$ and (b) selection intensity is strong $\left(\omega^{2}=5\right)$ following different initial states. Divergent selection was preceded by one of three different initial states: neutral mutation-migration-drift equilibrium (solid line, scenarios 4 and 2), an initial phase of 100 generations of uniform selection (dashed line, scenarios 6 and 5) or an initial phase of 100 generations of weakly divergent selection (dotted line, scenarios 8 and 7). The lines represent the mean of 50 stochastic replicates, and the number of loci is 10 .

difference in allele frequency between populations. As can be seen on Figure $3, V_{\mathrm{B}}$ does not reach $\operatorname{Var} Z_{\mathrm{OPT}}$ (the variance of optimal values within populations according to stabilizing selection). The maximum value of the $V_{\mathrm{B}} / \operatorname{Var} Z_{\mathrm{OPT}}$ ratio was a function of the strength of stabilizing selection and did not vary with the number of loci: the mean value obtained was 0.67 when $\omega^{2}=50$ and 0.94 when $\omega^{2}=5$. Under extensive gene flow $(\mathrm{Nm}=10), \mathrm{Var} Z_{\mathrm{OPT}}$ can be reached only if stabilizing selection is strong while under weak selection, the final $V_{\mathrm{B}}$ value is less than half of $\operatorname{Var} Z_{\mathrm{OPT}}$ (Supplementary Table 1). No changes to the transient dynamics were observed when the number of loci was changed to 5 or 30 (data not shown). The only change was the overall higher values of $\theta_{\mathrm{W}}$ and $\theta_{\mathrm{B}}$ when the number of loci increased.

The general trends of variation of $\theta_{\mathrm{B}}$ were maintained when the starting conditions of simulations were modified. Indeed, regardless whether populations were at mutation-migration-drift equilibrium, undergoing uniform selection or moderate divergent selection, the responses of $\theta_{\mathrm{B}}$ to the induction of strong divergent selection was a very rapid and steep increase (Figures $4 \mathrm{a}$ and $\mathrm{b}$ ). The steepness of the response is mainly dependent on the strength of the ongoing stabilizing selection, and not on the initial conditions. However, the range of increase of $\theta_{\mathrm{B}}$ was larger when uniform selection was the starting point $\left(\theta_{\mathrm{B}}\right.$ negative at generation 0$)$, because the lag between the imposed variance of optimal values $\left(\operatorname{Var} Z_{\mathrm{OPT}}\right)$ and the between-population variance $\left(V_{\mathrm{B}}\right)$ at generation 0 was much larger than under other starting conditions. $\theta_{\mathrm{B}}$ is the immediate response to compensate the 
lag. We also monitored the changes in $\theta_{\mathrm{B}}$ when the number of loci was 5 and 30. There was no change in the dynamics, but the values after 100 generations differed according to the theoretical predictions (Equation (12)): they ranged from 1.76 to 2.11 with 5 loci, from 3.17 to 4.15 with 10 loci and from 7.74 to 8.36 with 30 loci.

\section{Dynamics of $G_{\mathrm{ST} q}$ and $Q_{\mathrm{ST}}$}

As for of $\theta_{\mathrm{W}}$ and $\theta_{\mathrm{B}}$, we monitored first the variation of $Q_{\mathrm{ST}}$ and $G_{\mathrm{ST} q}$ under scenarios 1-4 following an initial mutation-migration-drift equilibrium (Figure 5). There are striking differences between the variation of $Q_{\mathrm{ST}}$ and $G_{\mathrm{ST} q}$ on the one hand and $\theta_{\mathrm{W}}$ and $\theta_{\mathrm{B}}$ on the other. $\theta_{\mathrm{W}}$ and $\theta_{\mathrm{B}}$ reach minimum and maximum values during the first few generations, whereas $Q_{\mathrm{ST}}$ and, especially $G_{\mathrm{ST} q}$, increases more steadily. The main driver of $G_{S T q}$ appears to be the strength of stabilizing selection, which opposes the effect of gene flow, as described above.

Throughout the selection scenario, $G_{\mathrm{ST} q}$ remained lower than $Q_{\mathrm{ST}}$, as a straightforward consequence of $\theta_{\mathrm{W}}$ being lower than $\theta_{\mathrm{B}}$ (inequalities 6). $Q_{S T}$ increased very rapidly in the first few generations, with a steeper slope under strong stabilizing selection. Subsequently, the dynamics of $Q_{\mathrm{ST}}$ followed that of $G_{\mathrm{ST} q}$, with a steady increase when stabilizing selection was strong (Figures $5 \mathrm{c}$ and d), and a smoother or flat course when stabilizing selection was weak (Figures $5 \mathrm{a}$ and $\mathrm{b}$ ). The decoupling of $Q_{\mathrm{ST}}$ and $G_{\mathrm{ST} q}$ was not changed when initial conditions were either uniform selection or moderate divergent selection (Figures $5 \mathrm{~b}, \mathrm{~d}$ and $6 \mathrm{a}, \mathrm{b}$ ). Whether $Q_{\mathrm{ST}}$ is 0 or already higher than the neutral expectation at generation 0 , the time trends during the first 100 generations are similar. The same conclusions hold also for $G_{\mathrm{ST} q}$. Particularly, the very rapid increase of $Q_{\mathrm{ST}}$ during the early generations is created by the steep variation of $\theta_{\mathrm{B}}$, being the main driver of $Q_{\text {ST }}$.

The patterns depicted in Figures 2-6 indicate that the variation of population differentiation induced by selection follows two phases. A detailed dissection of the early phase (comparison of Figures 3 and 5 with Figure 2) suggested that the main driver of the rapid increase in both $V_{\mathrm{B}}$ and $Q_{\mathrm{ST}}$ was the variation of $\theta_{\mathrm{B}}$, rather than that of $G_{\mathrm{ST} q}$. Interestingly, $\theta_{\mathrm{B}}$ may reach even higher values when the trait is controlled by a larger number of loci (data not shown, but see asymptotic values in Table $2 \mathrm{~b}$ ), enhancing the capture of intergenic disequilibria, as the larger number of loci provides a larger number of opportunities for favorable allelic associations. The dynamics during this early phase is highly analogous to that of the genetic variance of a trait undergoing selection within a single population (Bulmer, 1974, 1980). Indeed, Bulmer indicated that allele frequencies probably remain constant over the first few generations of selection, whereas the genetic variance of the trait decreases, due mostly to negative intergenic linkage disequilibrium. In our multiple-population context, the Bulmer effect may be thus generalized as follows: the short-term response to selection is characterized by a decrease in within-population variance mediated by negative values of $\theta_{\mathrm{W}}$ in each population on one the hand, and an increase in between-population variance mediated by a rapid increase in positive $\theta_{\mathrm{B}}$ values on the other hand. This early phase is characterized by the rapid building up of the covariance of allelic effects, but allelic frequencies within populations change very slowly. The increase in covariance is followed by an increase in between-population variance, $V_{\mathrm{B}}$, which peaks at the end of the first phase. As this point, populations are locally adapted (that is, the between-population variance is closest to the optimal variance). The duration of the early phase varies from a little over 10 to 30 generations when local stabilizing selection is strong, and from 50 to 80 generations when local stabilizing selection is weak .In the second
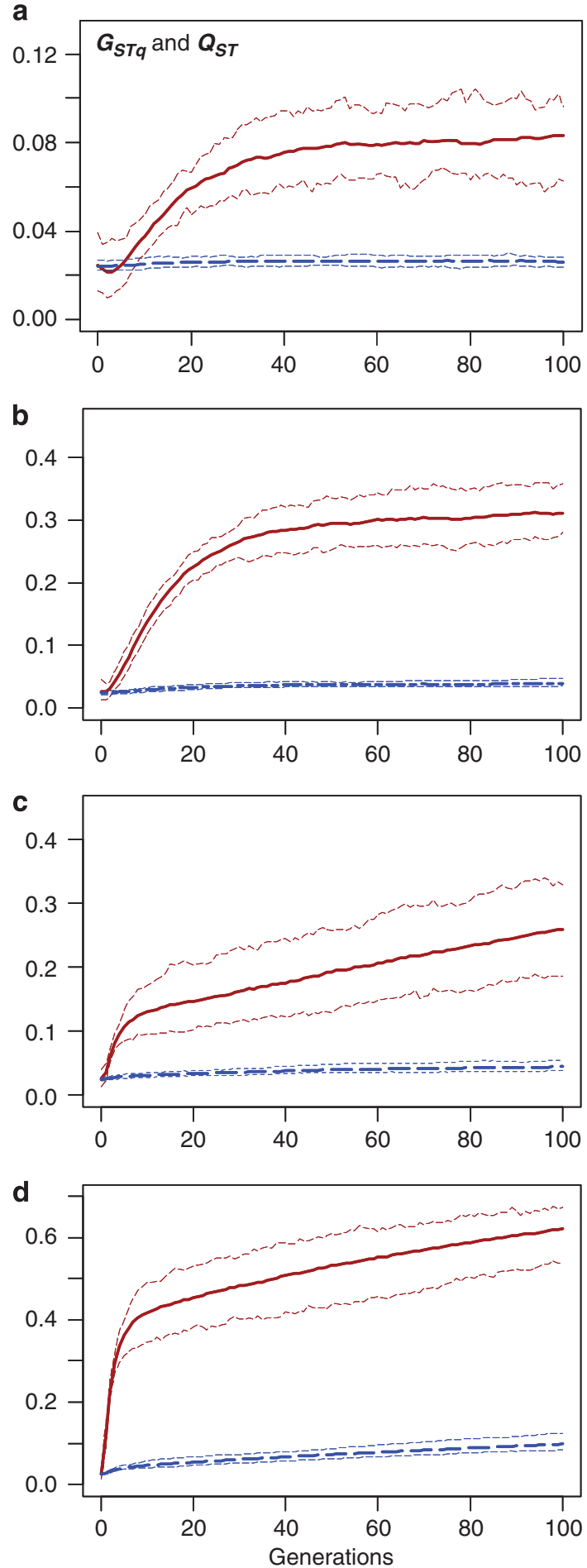

Figure 5 Dynamics of $G_{\mathrm{ST} q}$ (in blue) and $Q_{\mathrm{ST}}$ (in dark red) over successive generations in response to different selection scenarios in the case of 10 loci contributing to the trait. (a) Scenario $3, \omega^{2}=50$ and $\operatorname{Var} Z_{\mathrm{OPT}}=1$, weak stabilizing and moderate divergent selection, (b) scenario $4, \omega^{2}=50$ and $\operatorname{Var} Z_{\mathrm{OPT}}=5$, weak stabilizing and strong divergent selection, (c) scenario 1 , $\omega^{2}=5$ and $\operatorname{Var} Z_{\mathrm{OPT}}=1$, strong stabilizing and moderate divergent selection, (d) scenario $2, \omega^{2}=5$ and $\operatorname{Var} Z_{\mathrm{OPT}}=5$, strong stabilizing and strong divergent selection, 50 repetitions of simulations were conducted for each scenario, bold lines indicate mean values and dashed lines encompass $95 \%$ of the values. 
a

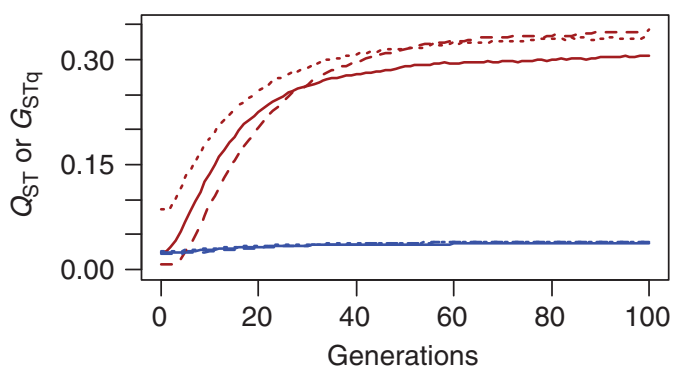

b

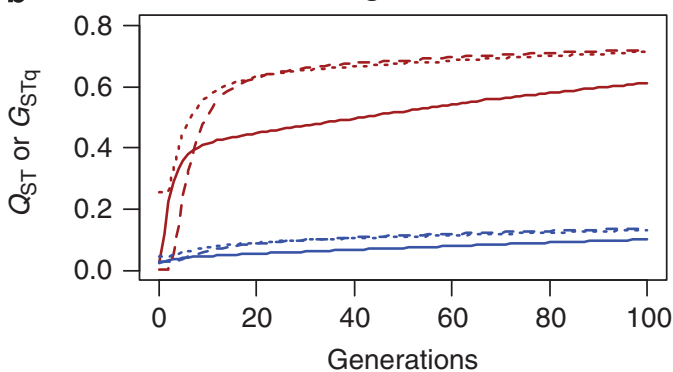

Figure 6 Response of $Q_{S T}$ (red lines) and $G_{S T q}$ (blue lines) to divergent selection $\left(\operatorname{Var} Z_{\mathrm{OPT}}=5\right)$ when (a) selection intensity is weak $\left(\omega^{2}=50\right)$ and $(\mathbf{b})$ selection intensity is strong $\left(\omega^{2}=5\right)$. Divergent selection was preceded by one of three different initial states: neutral mutation-migration-drift equilibrium (solid line, scenarios 4 and 2), an initial phase of 100 generations of uniform selection (dashed line, scenarios 6 and 5) or an initial phase of 100 generations of weakly divergent selection (dotted line, scenarios 8 and 7). The lines represent the mean of 50 stochastic replicates, and the number of loci is 10 .

phase, there is a slower steady increase in $Q_{\mathrm{ST}}$ driven by $G_{\mathrm{ST} q}$, with no further increase in $\theta_{\mathrm{B}}$. $\theta_{\mathrm{B}}$ remains larger than $\theta_{\mathrm{W}}$, but differences in allelic frequencies gradually increase, contributing to the steady increase in $Q_{\mathrm{ST}}$.

Changes of selection regimes and decoupling of $G_{\mathrm{ST} q}$ and $Q_{\mathrm{ST}}$ An interesting extension of the comparative in silico monitoring of $G_{\mathrm{ST} q}$ and $Q_{\mathrm{ST}}$ is their response to environmental changes, which can be provoked by changing $Z_{\mathrm{OPT}}$ values assigned to the different populations. To do so, we compared two symmetric scenarios: divergent selection to uniform selection (scenarios 5 and 6) versus uniform to divergent selection (scenarios 9 and 10; Figures 7 and 8). The rapidity of the response of $\theta_{\mathrm{B}}$ after generation 100 was very similar, whether the level of divergent selection was increasing or decreasing, resulting in almost a symmetric pattern (Figure 7). The same conclusions can be drawn for $Q_{S T}$, whereas the changes of selection regimes have only minor impact on $G_{S T q}$ (Figure 8 ). The same simulations were conducted under moderate divergent selection during phase B (scenario 11 and 12) and by changing the number of loci to 5 and 30, but the dynamics of $G_{\mathrm{ST} q}$ and $Q_{\mathrm{ST}}$ following the changing of selection regimes were very similar to those observed in Figure 8 (data not shown).

\section{DISCUSSION}

Evidence for the decoupling of differentiation between traits and genes

Our results support earlier findings suggesting that substantial decoupling may occur between $Q_{\mathrm{ST}}$ and $G_{\mathrm{ST} q}$ (Latta, 1998, 2004; McKay and
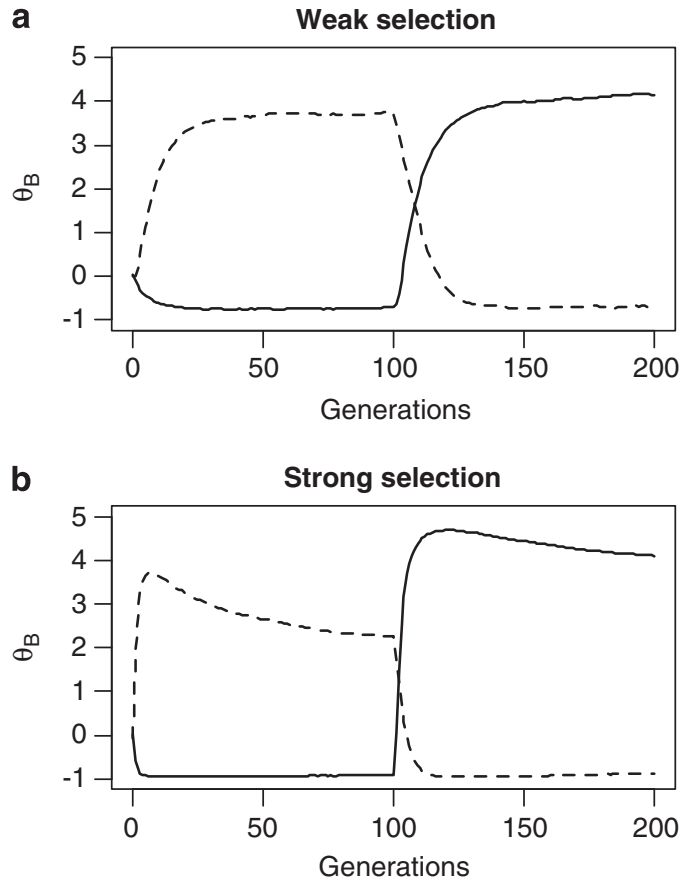

Figure 7 Response of $\theta_{\mathrm{B}}$ to changes of divergent selection regimes. (a) Weak stabilizing selection during phases $A$ and $B$. Solid line: transition from uniform to divergent selection (scenario 6). Dashed line: transition from divergent to uniform selection (scenario 10). (b) Strong stabilizing selection during phases $A$ and $B$. Solid line: transition from uniform to divergent selection (scenario 5). Dashed line: transition from divergent to uniform selection (scenario 9), 50 repetitions of simulations were conducted for each scenario. Lines represent mean values of the repetitions.

Latta, 2002). We explored in greater detail the evolutionary factors enhancing decoupling and showed that strong divergent selection and strong selection intensity within populations were the predominant drivers of the lag between $Q_{S T}$ and $G_{S T q}$. Furthermore, this discrepancy increases as the number of loci involved in the trait increases. The conclusions drawn from theoretical analyses can be compared with experimental estimates of $Q_{\mathrm{ST}}$ and $G_{\mathrm{ST} q}$. The genes involved in quantitative traits have not yet been fully identified, but candidate genes have been identified for various traits of adaptive significance, not only in model species. A few recent studies have compared $Q_{\text {ST }}$ with $G_{\mathrm{ST} q}$ estimates for candidate genes with $G_{\mathrm{ST}}$ for neutral markers. The traits of interest investigated were resistance to drought stress in Pinus pinaster (Eveno et al., 2008), bud set in P. sylvestris (Pyhäjärvi et al., 2008) and Picea abies (Heuertz et al., 2006), phenological traits (bud burst, length of growing season and leaf abscission) and growth traits (height growth and diameter) in Populus tremula (Hall et al., 2007 and Luquez et al., 2007), bud burst in Quercus petraea (Derory et al., 2010) and growth, phenology and wood characters in Picea glauca (Namroud et al., 2008). In each case, the level of differentiation of the candidate genes was similar to that of neutral markers, whereas $Q_{\mathrm{ST}}$ values were much higher. The selection of candidate genes may not have been stringent enough to identify the only genes causally involved in expression of the trait of interest, but the concordant results obtained in the various studies carried out support our theoretical predictions that differentiation at individual genes may be limited, even for traits displaying high levels of differentiation in common garden experiments. We also suspect that this may be the case in general for trees and other species with 

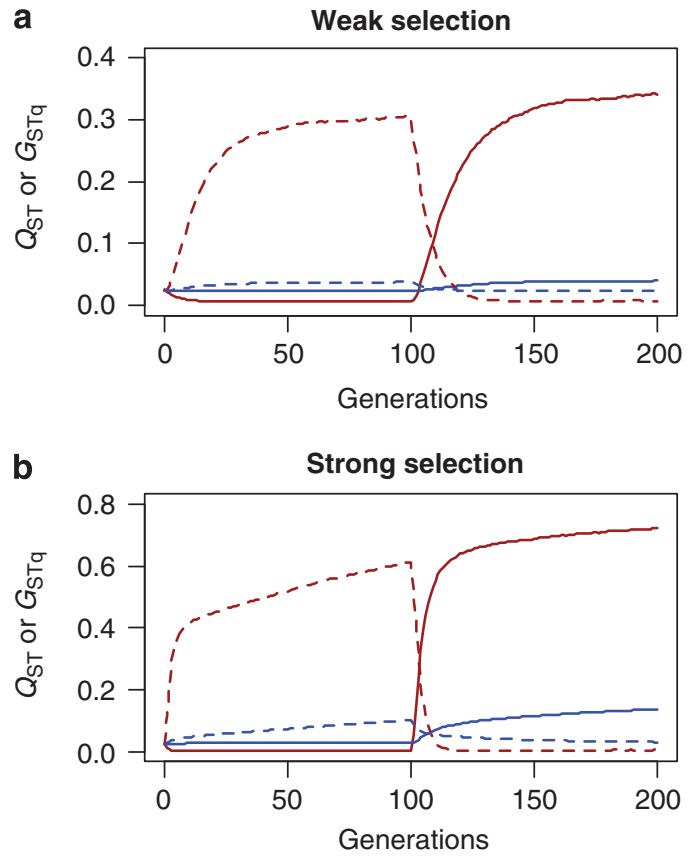

Figure 8 Response of $Q_{S T}$ (red lines) and $G_{S T q}$ (blue lines) to changes of divergent selection regimes. (a) Weak stabilizing selection during phases $A$ and B. Solid line: transition from uniform to divergent selection (scenario 6). Dashed line: transition from divergent to uniform selection (scenario 10). (b) Strong stabilizing selection during phases A and B. Solid line: transition from uniform to divergent selection (scenario 5). Dashed line: transition from divergent to uniform selection (scenario 9), 50 repetitions of simulations were conducted for each scenario. Lines represent mean values of the repetitions.

large populations, high levels of gene flow and strong divergent selection.

\section{Transient dynamics of population differentiation}

Over and above the simple decoupling of $Q_{\mathrm{ST}}$ and $G_{\mathrm{ST} q}$, our results highlight the striking differences in the dynamics of these two differentiation measurements, once selection has been established. The differences observed in the variation of $\theta_{\mathrm{W}}$ and $\theta_{\mathrm{B}}$, and of $Q_{\mathrm{ST}}$ and $G_{\mathrm{ST} q}$ over successive generations (illustrated in Figures 3-8) highlight the differences in trait and gene divergence as a result of selection. The dynamics can clearly be separated into two phases. Very early in the first phase, $\theta_{\mathrm{W}}$ and $\theta_{\mathrm{B}}$ follow a bell-shaped curve, reaching a maximum for $\theta_{\mathrm{B}}$ (or a minimum for $\theta_{\mathrm{W}}$ ) after $<20$ generations. The increase in $\theta_{\mathrm{B}}$ is followed by an increase in between-population variance $\left(V_{\mathrm{B}}\right)$, which peaks at 10-30 generations when local stabilizing selection is strong, and at $50-80$ generations when local stabilizing selection is weak. During this period of increase, $Q_{\mathrm{ST}}$ increases very rapidly, whereas $G_{\mathrm{ST} q}$ displays a much slower monotone increase. The second phase starts after $V_{\mathrm{B}}$ has reached its maximum value, and is characterized by a steady increase in $G_{\mathrm{ST} q}$. During this second phase, $Q_{\mathrm{ST}}$ increases more slowly, driven mostly by $G_{\mathrm{ST} q}$.

Slight variations of these general patterns were observed for the different selection scenarios, but the overall trends remained the same. In more biological terms, these patterns suggest two important conclusions: (1) that divergent selection first captures beneficial allelic associations at different loci in different populations and then targets changes in allelic frequencies and (2) that allelic associations promote rapid genetic divergence between populations more efficiently than changes in allelic frequencies.

In terms of evolution, this has the immediate consequence of very rapid differentiation at the trait level, once selection is established. The rapid response of $Q_{\mathrm{ST}}$ results from the building up of covariances between allelic effects within and between populations. $\theta_{\mathrm{W}}$ decays rapidly because of recombination, whereas $\theta_{\mathrm{B}}$ becomes the main driver of $Q_{\mathrm{ST}}$ during the first phase. In their review, Reznick and Ghalambor (2001) identified two principal contexts in which rapid evolution has been reported: new environments recently colonized by new populations and metapopulation structures in heterogeneous environments. In both scenarios, there are recent and new selection pressures, resulting in evolution during these early stages. These conditions match our scenario, in which populations rapidly differentiate. Most authors have tended to conclude that rapid evolution results from drastic demographic changes associated with the changes to the environment. However, our results suggest that there may also be a rapid accumulation of beneficial allelic associations. Several examples supporting rapid differentiation of traits have been reported in European tree species. Norway spruce displays the most recent wave of migration in Europe, spreading throughout southern Scandinavia over the last 2000 years by natural means or through human-mediated dispersion (Bradshaw and Lindbladh, 2005). Common garden experiments established with populations originating from these regions have shown extensive population differentiation for all traits assessed (Hannerz and Westin, 2000; Danusevicius and Gabrilavicius, 2001). Over more recent time scales, the introduction of northern red oak (Quercus rubra) into Europe over the last two centuries has been followed by the rapid divergence of the source population from the natural distribution and the introduced populations (Daubree and Kremer, 1993).

Our results have peculiar relevance in the context of climate change. Although we investigated responses to sudden changes of divergent selection in our simulations, we showed that allelic associations were immediately modified. Whether the transition was from moderate to strong divergent selection or from uniform to strong, the response elapsed during less than 10-20 generations. The lag of the response of $\theta_{\mathrm{B}}$ was slightly lower under the former than the latter transition. We suspect that under more gradual changes of environmental conditions, allelic associations would be changed during a shorter period. Evolutionary response to fill the lag between new optimal values induced by climate change and extant values of populations has triggered theoretical research on adaptation to climate change (Lande and Shannon, 1996; Burger and Krall, 2004). Our results suggest that the multilocus structure of complex traits and intergenic disequilibria should be considered in predictive models of adaptive divergence.

\section{Detection of selection signatures at the molecular level}

We showed that the decoupling of $Q_{\mathrm{ST}}$ and $G_{\mathrm{ST} q}$ is generated by the discrepancy between allelic covariances both within and between populations. At the molecular level, the signature of selection is therefore more visible on multilocus than on monolocus structures of diversity. At worst, during the early phase of selection, there may be no visible trace of selection at single loci, whereas strong correlations between allelic effects are expected. However, these conclusions must be considered with caution, as our analysis at the monolocus level is limited to the mean differentiation across all loci involved in the trait. We previously showed that $G_{\mathrm{ST} q}$ values may differ between loci and that heterogeneity increases with gene flow and low stabilizing selection (Le Corre and Kremer, 2003). Under such circumstances, the detection of outlier loci by genome scans would be successful, but only 
a small subset of genes would be identified. However, selection imprints can be detected at the molecular level by another method, based on the correlation of allelic effects between populations $\left(\theta_{\mathrm{B}}\right)$. It is difficult to estimate allelic effects, but the correlation of allelic frequencies may be used as a surrogate for $\theta_{\mathrm{B}}$. Correlations between allelic frequencies can be estimated from gene diversity surveys in natural populations, such as $G_{\mathrm{ST} q}$. In this respect, an appropriate initial approach would be to estimate the correlation between outlier loci detected on genome scans. Alternatively, multilocus measurements of population differentiation taking into account disequilibrium between alleles could also be used to detect multilocus structures (Kremer et al., 1997). However, these approaches may be tainted by confounding effects, generating covariances of allelic frequencies, such as demographic or historical effects. One highly predictable confounding effect may result from isolation by distance, and will affect all loci including those involved in the trait of interest. Divergent selection may follow continuous geographic gradients, resulting in an increase in $\operatorname{Var} Z_{\mathrm{OPT}}$ along the gradient. Covariances of allelic effects may therefore be generated independently by two different mechanisms. The second constraint on explorations of $\theta_{\mathrm{B}}$ or its surrogates is the very high degree of variation observed between repeats of the same scenario, suggesting the existence of a very high associated stochastic evolutionary variance (Figure 1).

\section{Concluding remarks}

In contrast to our earlier comparison between $Q_{\mathrm{ST}}$ and $G_{\mathrm{ST} q}$ this review is purposely limited to outcrossing species that exhibit large population sizes and extensive gene flow such as forest trees. Indeed, the built up of substantial population differentiation for fitness-related traits that is observed in provenance tests (common garden experiments) in the context of large pollen flow has not been elucidated so far. Here, we show that the pace at which allelic associations are generated can be responsible for the level of divergence observed in extant populations. We suspect that the building up of allelic associations is enhanced by the very large genetic diversity residing within populations, which is sustained by pollen flow. These results need, however, to be confirmed or refined in a more general context beyond the single trait approach and by considering explicitly the contribution of seed versus pollen to gene flow.

\section{CONFLICT OF INTEREST}

The authors declare no conflict of interest.

\section{ACKNOWLEDGEMENTS}

This research was supported by the European Commission through the directorate General Research within the fifth and sixth framework programmes (Research project TREEESNIPS (QLK3-CT-2002-01973) and Network of Excellence EVOLTREE (FP6 No. 016322)). We are grateful to two anonymous reviewers for their very helpful comments and suggestions.

Aitken SN, Yeaman S, Holliday JA, Wang T, Curtis-McLane S (2008). Adaptation, migration or extirpation: climatic changes outcomes for tree populations. Evol App/ 1: 95-111.

Beaumont MA, Balding JB (2004). Identifying adaptive genetic divergence among populations from genome scans. Mol Ecol 13: 969-980.

Björklund M, Ranta E, Kaitala V, Bach LA, Lundberg P, Stenseth NC (2009). Quantitative trait evolution and environmental change. PLOS One 4: e4521.

Bradshaw RHW, Lindbladh M (2005). Regional spread and stand-scale establishment of Fagus sylvatica and Picea abies in Scandinavia. Ecology 86: 1679-1686

Burger R, Krall C (2004). Quantitative-genetic models and changing environments. In Ferriere R, Dieckmann U, Couvet D (eds). Evolutionary Conservation Biology. Cambridge University Press: Cambridge, UK, pp 171-187.
Bulmer MG (1974). Linkage disequilibrium and genetic variability. Genet Res 23: 281-289.

Bulmer MG (1980). The Mathematical Theory of Quantitative Genetics. Clarendon Press: Oxford, 254p.

Crispo $E$ (2008). Modifying effects of phenotypic plasticity on interactions among natural selection, adaptation and gene flow. J Evol Biol 21: 1460-1469.

Danusevicius D, Gabrilavicius R (2001). Variation in juvenile growth rhythm among Picea abies provenances from the Baltic States and the adjacent regions. Scand J For Res 16: 305-317.

Daubree JB, Kremer A (1993). Genetic and phenological differentiation between introduced and natural populations of Quercus rubra L. Annales des Sciences Forestieres $\mathbf{5 0}$ (Suppl 1): 271s-280s.

Derory J, Scotti-Saintagne C, Bertocchi E, Le Dantec L, Graignic N, Jauffres A et al. (2010). Contrasting correlations between diversity of candidate genes and variation of bud burst in natural and segregating populations of European oaks. Heredity 104: 438-448.

Eveno E, Collada C, Guevara MA, Léger V, Soto A, Díaz L et al. (2008). Contrasting patterns of selection at Pinus pinaster Ait. drought stress candidate genes as revealed by genetic differentiation analyses. Mol Biol Evol 25: 417-437.

Ewens W (1972). The sampling theory of selectively neutral alleles. Theor Popul Biol 3 : 87-112.

Goudet J, Büchi L (2006). The effects of dominance, regular inbreeding and sampling design on Qst, an estimator of population differentiation for quantitative traits. Genetics 172: $1337-1347$.

Goudet J, Martin G (2007). Under neutrality, Qst > = Fst when there is dominance in an island model. Genetics 176: 1371-1374.

Hall D, Luquez V, Garcia VM, St Onge KR, Jansson S, Ingvarsson PK (2007). Adaptive population differentiation in phenology across a latitudinal gradient in European Aspen (Populus tremula, L.): a comparison of neutral markers, candidate genes, and phenotypic traits. Evolution 61: 2849-2860.

Hamilton DC, Cole DEC (2004). Standardizing a composite measure of linkage disequilibrium. Ann Hum Genet 68: 234-239.

Hannerz M, Westin J (2000). Growth cessation and autumn-frost hardiness in one-year-old Picea abies progenies from seed orchards and natural stands. Scand J For Res 15: 309-317.

Hendry AP, Day T, Taylor EB (2001). Population mixing and the adaptive divergence of quantitative traits in discrete populations: a theoretical framework for empirical tests. Evolution 55: 459-466.

Heuertz M, De Paoli E, Källmann T, Larsson H, Jurman I, Morgante M et al. (2006). Multilocus patterns of nucleotide diversity, linkage disequilibrium, and demographic history of Norway spruce. Genetics 174: 2095-2105.

Holderegger R, Herrmann D, Poncet B, Gugerli F, Thuiller W, Taberlet P et al. (2008). Land ahead: using genome scans to identify molecular markers of adaptive relevance. Plant Ecol Divers 1: 273-283.

Kapralov MV, Filatov DA (2006). Molecular adaptation during adaptive radiation in the Hawaiian endemic genus Schiedea. Plos One 1: e8.

Kawecki TJ (2008). Adaptation to marginal habitats. Annu Rev Ecol Evol Syst 39: 321-342.

Kirkpatrick M, Johnson T, Barton N (2002). General models of multilocus evolution. Genetics 161: 1727-1750.

König AO (2005). Provenance research: evaluating the spatial pattern of genetic variation. In: Geburek Th, Turok J (eds). Conservation and Management of Forest Genetic Resources in Europe. Arbora Publishers: Zvolen, Slovakia, pp 275-328.

Kremer A (1994). Diversité génétique et variabilité des caractères phénotypiques chez les arbres forestiers. Genet Sel Evol 26 (Suppl 1): 105s-123s.

Kremer A, Zanetto A, Ducousso A (1997). Multilocus and multitrait measures of differentiation for gene markers and phenotypic traits. Genetics 145: 1229-1241.

Lande R, Shannon S (1996). The role of genetic variation in adaptation and population persistence in a changing environment. Evolution 50: 434-437.

Latta RG (1998). Differentiation of allelic frequencies at quantitative trait loci affecting locally adaptive traits. Am Nat 151: 283-292.

Latta RG (2004). Gene flow, adaptive population divergence and comparative population structure across loci. New Phytol 161: 51-58.

Le Corre V, Kremer A (2003). Genetic variability at neutral markers, quantitative trait loci and trait in a subdivided population under selection. Genetics 164: 2005-2019.

Lopez S, Rousset F, Shaw FH, Shaw RG, Ronce O (2008). Migration load in plants: role of pollen and seed dispersal in heterogeneous landscapes. J Evol Biol 21: 294-309.

Luquez V, Hall D, Albrectsen B, Karlsson J, Ingvarsson PK, Jansson S (2007). Natural phenological variation in aspen (Populus tremula): the Swedish Aspen Collection. Tree Genet Genome 4: 279-292.

McKay JK, Latta RG (2002). Adaptive population divergence: markers, QTLs and traits. Trends Ecol Evol 17: 185-291.

Morgenstern EK (1996). Geographic Variation in Forest Trees. University of British Columbia Press: Vancouver, Canada, 209p.

Namroud MC, Beaulieu J, Juge N, Laroche J, Bousquet J (2008). Scanning the genome for single nucleotide polymorphisms involved in adaptive population differentiation in white spruce. Mol Ecol 17: 3599-3613.

Nei M (1987). Molecular Evolutionary Genetics. Columbia University press: New York, $512 \mathrm{p}$.

Nei M, Li WH (1973). Linkage disequilibrium in subdivided populations. Genetics 75 : 213-219.

Nielsen R (2005). Molecular signatures of natural selection. Ann Rev Genet 39: 197-218. 
Nosil P, Funk DJ, Ortiz-Barrientos D (2009). Divergent selection and heterogeneous genomic divergence. Mol Ecol 18: 375-402.

Pyhäjärvi T, García-Gil R, Knürr T, Mikkonen M, Wachowiak W, Savolainen O (2008). Demographic history has influenced nucleotide diversity in European Pinus sylvestris populations. Genetics 177: 1713-1724.

Rasanen K, Hendry AP (2008). Disentangling interactions between adaptive divergence and gene flow when ecology drives diversification. Ecol Lett 11: 624-636.

Reznick DN, Ghalambor CK (2001). The population ecology of contemporary adaptations: what empirical studies reveal about the conditions that promote adaptive evolution. Genetica 112-113: 183-198.

Roff DA (2002). Life History Evolution. Sinauer Associates: Sunderland Massachusetts.
Santure AW, Wang J (2009). The joint effects of selection and dominance on the Qst -Fst contrast. Genetics 181: 259-276.

Savolainen 0, Pyhajarvi T, Knurr T (2007). Gene flow and local adaptation in trees. Annu Rev Ecol Evol Syst 38: 595-619.

Storz JF (2005). Using genome scans of DNA polymorphism to infer adaptive population divergence. Mol Ecol 14: 671-688.

Weir BS (1990). Genetic Data Analysis. Sinauer Associates: Sunderland, MA, 377p.

Wright JW (1976). Introduction to Forest Genetics. Academic Press: New York, NY, USA.

Wright SI, Gaut BS (2006). Molecular population genetics and the search for adaptive evolution in plants. Mol Biol Evol 22: 506-519.

Supplementary Information accompanies the paper on Heredity website (http://www.nature.com/hdy) 